\title{
OIL ASPIRATION PNEUMONIA IN INFANCY
}

\author{
BY \\ F. S. W. BRIMBLECOMBE, L. CROME, M.C., and J. P. M. TIZARD \\ From the Pathological and Paediatric Departments of St. Mary's Hospital, London
}

(Received for Publication June 21, 1950)

It has been recognized for over 20 years that the therapeutic administration of oily substances either nasally or orally may in certain circumstances lead to the development of pneumonia, often fatal in its outcome.

In 1940 Bishop collected and reviewed 136 cases, and in 1941 Kaplan stated that he had been able to find references to 411 cases in the literature, but subsequent cases continue to be recorded. It is our intention, therefore, to review the information available and to describe a case which illustrates certain aetiological and pathological aspects of paraffin pneumonia.

\section{Historical}

The first description of oil aspiration pneumonia in animals was by Waters, Bayne-Jones, and Rowntree in 1917, who during the course of an investigation to decide the most suitable substances for bronchography, showed that bromoform in olive oil when injected into the trachea of a rabbit caused a fatal pneumonia.

Subsequent animal experiments by GuieyssePellissier in 1920 and by Corper and Freed in 1922 showed that both vegetable and mineral oils produced a characteristic type of pneumonic change when injected intratracheally into dogs and rabbits.

The original description of oil aspiration pneumonia in man was by Laughlen in 1925 who described four cases diagnosed at necropsy; three cases were in infants and one in an adult. In two cases oral medicinal paraffin was the oil incriminated and in two cases oily nasal drops.

Laughlen followed up this pathological finding by reproducing in experimental animals identical pulmonary changes at necropsy after the intratracheal injection of the same oils as had been given to his patients.

In 1927 Pinkerton reported six cases of oil aspiration pneumonia found at necropsy, comprising five infants and one 6-year-old boy. Five of these children had suffered from chronic illness for a considerable time before thei death. Two of the infants were described as always difficult to feed, another infant had convulsions due to a subdural haematoma discovered at the necropsy, and one case (Pinkerton's case 4) may well have been one of cystic fibrosis of the pancreas. The older boy was a tension athetoid. Pinkerton also showed that the identification of the oil in the lungs might be assisted by differential staining.

In 1928 Pinkerton reported the varying pulmonary changes produced by animal, vegetable, and mineral oils injected intratracheally in animals, noting how the pathological changes altered according to the time interval between the injection and the time of death, and also that damage to the lung was produced by animal and mineral oils, vegetable oils being relatively innocuous.

In 1932 Pierson recorded what appears to be the first diagnosis of oil aspiration pneumonia made during life. The diagnosis was suggested by the radiological picture which showed more abnormality than the clinical signs in the chest suggested.

In 1933 Fischer-Wasels showed that a more precise determination both of the nature of the aspirated oil and the amount present in the lungs could be obtained by extraction and physicochemical analysis.

A clear description of the pathological findings in the lungs was given by Ikeda in 1935, who described seven cases, all in children, diagnosed at necropsy.

In the great majority of cases, however, the exact nature and total amount of oil in the lungs has not been determined by extraction and by physicochemical analysis.

\section{Case History}

A boy aged $8 \frac{1}{2}$ months was brought to St. Mary's Hospital with the complaint that he had been losing weight for the previous three months and had suffered from stertorous breathing and excessive sweating for one month.

On being questioned his mother gave the following history. He was a first child, born weighing $7 \mathrm{lb}$. $(3 \cdot 2 \mathrm{~kg}$.) after a normal pregnancy. He was successfully breast-fed for three months when he was weaned on to a half-cream proprietary dried milk and given six feeds per day each 
containing $4 \mathrm{dr}$. of the milk powder in $4 \mathrm{oz}$. water. A few weeks later a full-cream dried milk was substituted and he was given five feeds a day each containing $4 \mathrm{dr}$. of the milk powder and one teaspoonful of sugar in $4 \mathrm{oz}$. of water. He was also given one teaspoonful of cod liver oil daily and unsweetened orange juice. On this seemingly inadequate diet he is said to have increased his weight to $18 \mathrm{lb} .11 \mathrm{oz}$. $\left(8.5 \mathrm{~kg}\right.$.) at $5 . \frac{1}{2}$ months. At that age his health, previously good, began to deteriorate. His parents attributed this to teething and in the course of two or three weeks gave him on nine occasions a proprietary teething powder which contains $0.75 \mathrm{gr}$. calomel.

His symptoms were limpness, vomiting, excessive perspiration, fretfulness, and loss of appetite. At 6 months, although not obviously photophobic, he began to rub his eyes and developed a bilateral conjunctival discharge which was treated with penicillin drops. It was also noticed at this time that his hands and, more especially, feet, appeared to itch and he would rub them together. Neither his hands nor feet were pink, but the feet felt cold and later skin peeled from between the fingers and toes. He appeared peculiarly lifeless and made no attempt to sit up or stand on his mother's lap as he had done previously. He also became constipated, hard stools being passed every other day, and so from the age of 6 months until his admission to hospital he was given three teaspoonfuls of medicinal paraffin daily. He never struggled when being dosed.

From $7 \frac{1}{2}$ months the sweating became yet more excessive and his mother noticed rapid noisy breathing, especially at night. He had no cough. Nose drops were not administered.

In the first month of his illness, that is from the age of $5 \frac{1}{2}$ months, he lost about $2 \mathrm{lb}$. $(0.91 \mathrm{~kg}$.) in weight; about half this was regained in the second month but a further loss occurred in the third.

$\mathrm{He}$ was never given more than $20 \mathrm{oz}$. per day of the full-cream milk (constituted as stated above) and the only additional food given was the daily dose of cod liver oil and the yolk of an egg every other day: Broth and groats were tried but were consistently refused.

On examination he appeared a thin, pale, listless infant and showed remarkable muscular hypotonia although he could move his limbs normally. Tendon reflexes were absent. He weighed $16 \mathrm{lb} .9 \mathrm{oz}$. (7.5 kg.). The temperature was normal, but the pulse rate was 130 per minute and the respiratory rate 60 per minute.

Small lymph nodes were palpable in the neck, axillae, and groins. The skin was excessively moist, but otherwise normal. The two lower central incisors had erupted. The pharynx was slightly inflamed. The thorax was somewhat barrel shaped with eversion of the rib margins, the movement was diminished on the right side, but there was otherwise no abnormal physical sign in the chest.

A radiograph of the chest (Fig. 1) showed ' diffuse parenchymal inflammatory changes of a pneumonic type in the right upper lobe and in the more medially lying segments of the right lower lobe'.

The infant was admitted to hospital and the following investigations were carried out. Nose and throat swabs were cultured but grew no pathogenic organisms. The cerebrospinal fluid, which was sterile, contained lymphocytes 1 per c.mm., protein $100 \mathrm{mg}$., chlorides $773 \mathrm{mg}$., and sugar $145 \mathrm{mg}$. per $100 \mathrm{ml}$.

On the day following his admission to hospital his condition suddenly worsened. His pulse rate rose to 200 per minute and his temperature to $101^{\circ} \mathrm{F}$. Respirations were rapid and shallow and the lower ribs were
Fig. 1.-Radiograph of chest on admission.

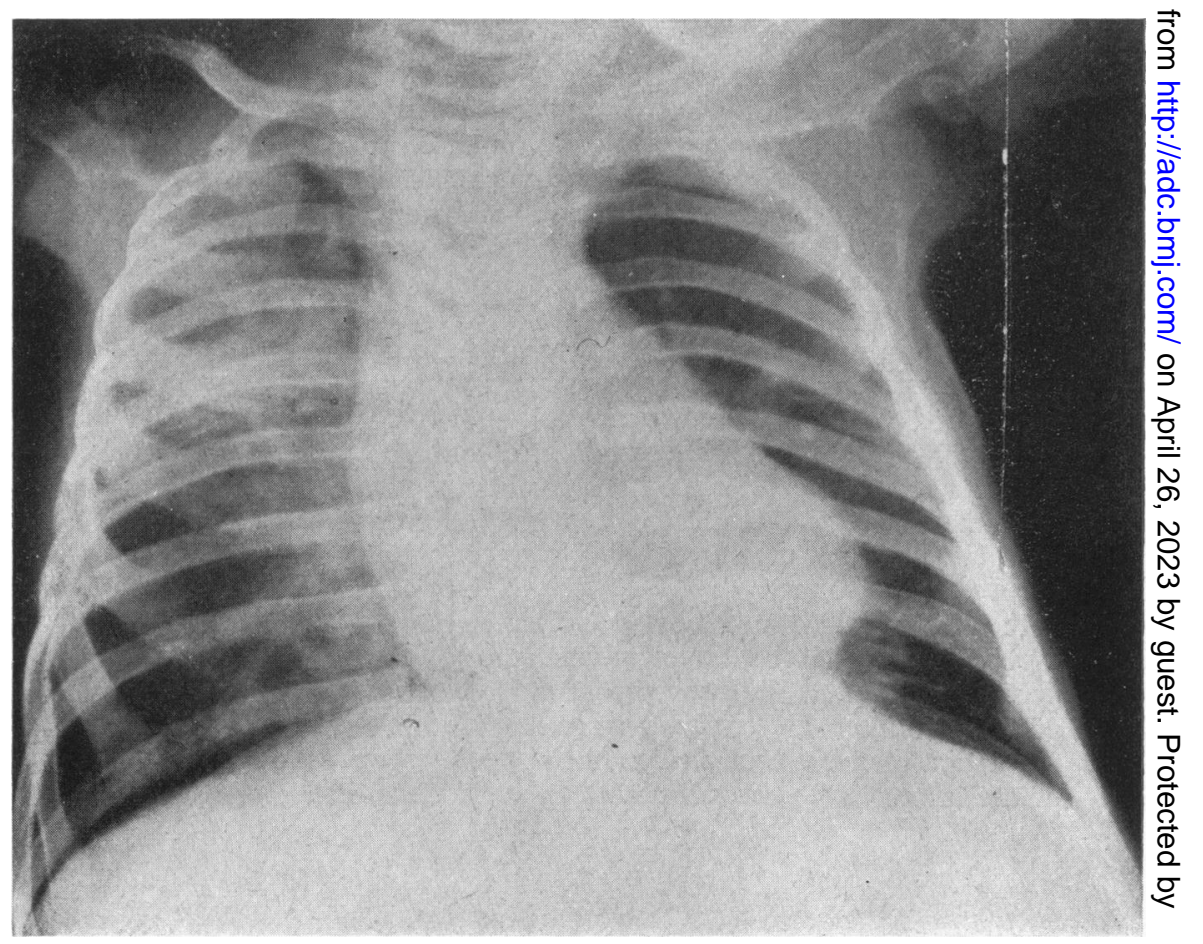




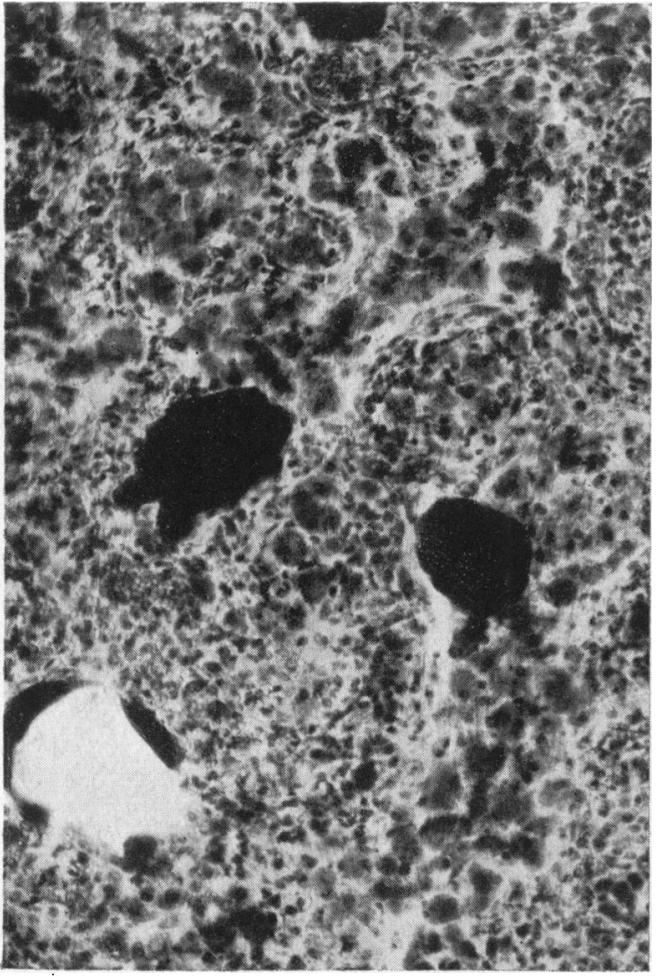

Fig. 4.-Photomicrograph of oil-laden macrophages in the alveoli and a bronchiole. Haematoxylin and eosin. $\times 200$.

FIG. 5.-Photomicrograph of coarse globules of oil in macrophages. Cellular exudate in the interalveolar septa. Haematoxylin and eosin. $\times 330$

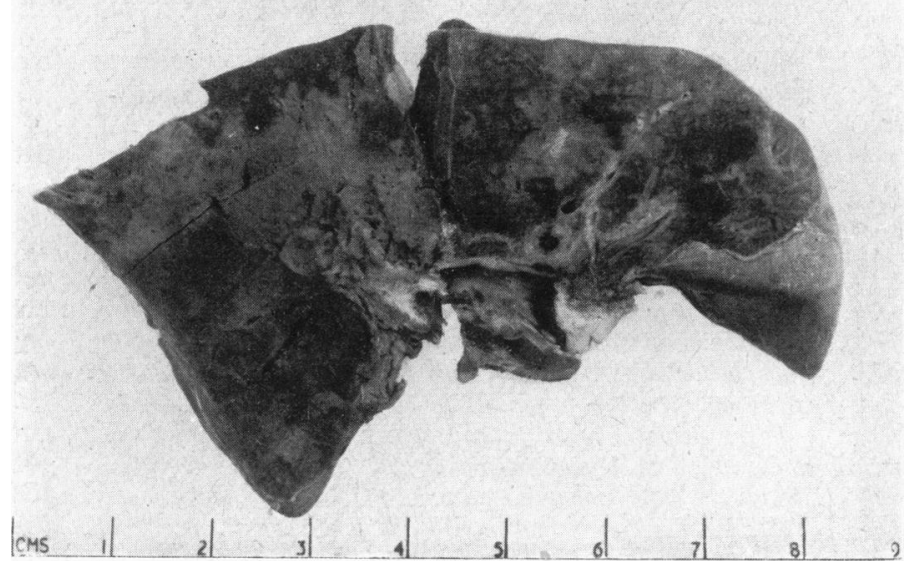

Fig. 2. Cut surface of the formalinized lung. There is complete consolidation with a pale, mottled, geographical pattern.

FIG. 3.-Photomicrograph showing large free globules of oil in the alveoli, which also contain numerous oil-laden macrophages. Some oil globules have fallen out leaving empty spaces. Frozen sections. Sudan III and haematoxylin. $\times 200$.
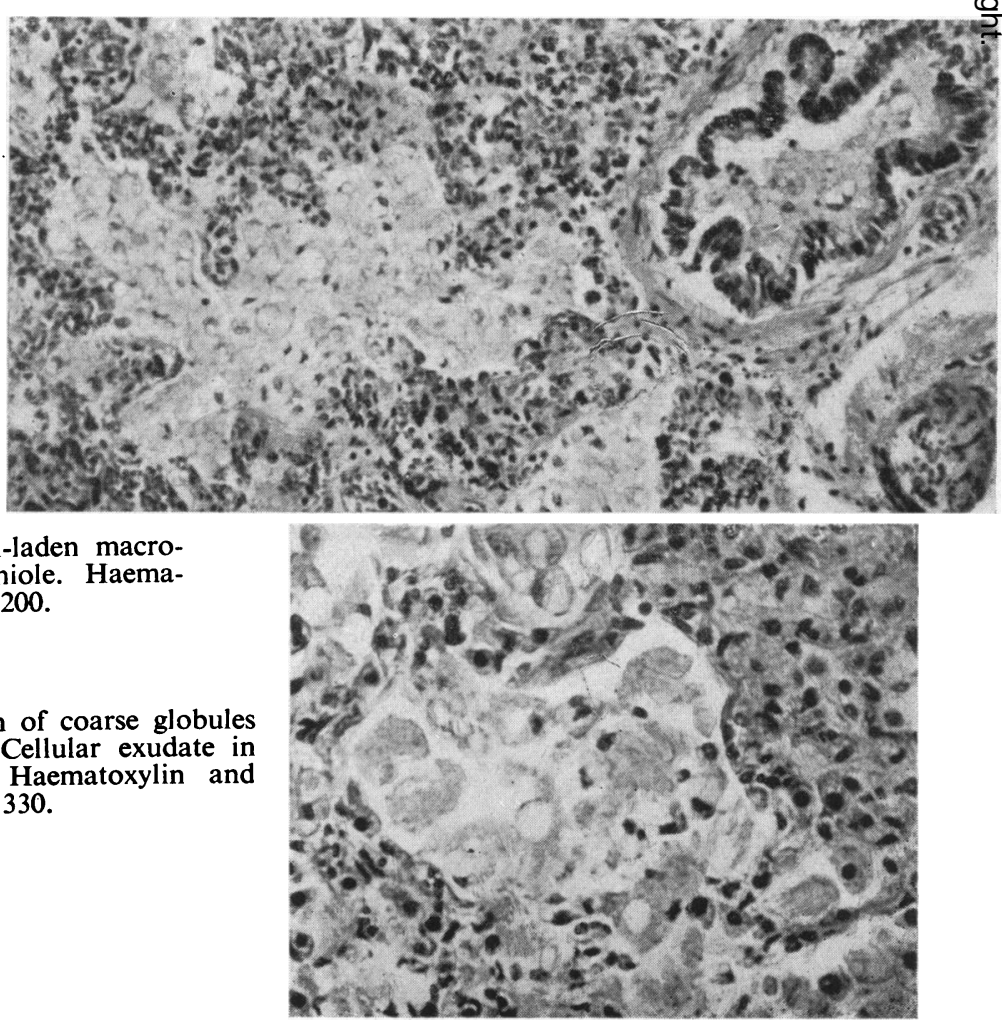
drawn in on inspiration. In a few hours the temperature rose to $106^{\circ}$, the infant became cyanosed, fell into coma and died.

The history suggested the diagnosis of pink disease, and the tachycardia, hypotonia, absence of deep reflexes, and excessive sweating found on examination were also in favour of this diagnosis. Whatever the cause of his anorexia, however, it resulted in the infant tolerating a diet quite inadequate for his age. This extreme underfeeding would account for the constipation which was treated with large doses of liquid paraffin over a prolonged period.

The possibility of lipoid pneumonia was not considered until the pcst-mortem examination. As his mother had been suspected of having pulmonary tuberculosis two years previously, primary intrathoracic tuberculosis was considered a possibility.

Necropsy. Necropsy was performed 11 hours after death. The subject was a male infant $27 \frac{1}{2}$ in. $(70 \mathrm{~cm}$.) in height and weighing $15 \mathrm{lb} .7 \mathrm{oz}$. (7 kg.). A small amount of fluid blood was present in the left ventricle; the other heart chambers were empty. The ductus arteriosus was closed. The foramen ovale was patent.

A thin film of oil was found covering the mucous surfaces of the trachea and bronchi and this gave the first indication of the true diagnosis. The lungs were pale, solid and firm, and each portion sank in water. Areas of greyish mottling were seen on the cut surface against the pale brown background of the lung. These areas were fairly evenly distributed throughout both lungs (Fig. 2). The pleura was smooth and glistening. When the lung was immersed in water minute globules of oil rose to the surface and coalesced to form a thin refractile film showing a play of colours.

The only other findings worthy of note were: the intestines were moderately distended. Peyer's patches were prominent in the ileum. Malpighian bodies were clearly visible on the surface of the spleen. A mild degree of phimosis was present. The testes were in the inguinal canals.

A culture taken from the lungs at necropsy grew a mixed flora of Staphylococcus aureus, Streptococcus viridans, non-haemolytic streptococci, and some diphtheroid bacilli.

Chemical Examination. The wet formalized lung weighed $121 \mathrm{~g}$. A representative portion weighing $7 \mathrm{~g}$. was treated as follows: (1) The tissue was homogenized in water in a Waring blender. (2) The emulsion was then treated repeatedly with ether which was subsequently evaporated. (3) The residue was saponified in $5 \%$ potassium hydroxide in alcohol. (4) The alcohol was removed by distillation. (5) The residue was extracted with ether and washed with water. (6) The extract was then dehydrated with anhydrous sodium sulphate and dried at $110^{\circ} \mathrm{C}$. for one hour. (7) The product was weighed and the paraffin separated from the steroids with petroleum ether. The weight of the product before separation was $0.834 \mathrm{~g}$. and after separation $0 \cdot 800$. The $7 \mathrm{~g}$. of wet lung therefore yielded approximately $11^{\circ}$ of paraffin by weight. This figure must be somewhat lower than the true paraffin content, on account of the unavoidable loss of oil during the handling of the material before extraction. The specific gravity of the paraffin was 0.85 , and it did not react with a mixture of sulphuric and fuming nitric acid. The spleen and liver treated similarly yielded no paraffin.

Histology. Paraffin-embedded sections were stained with Ehrlich's haematoxylin and eosin, iron haematoxylin and Van Gieson, Ziehl-Nielsen's stain and with Marchi's osmic acid stain. Frozen sections were examined unstained and were treated with absolute alcohol, xylol, and chloroform for fat solubility. They were also treated with varying dilutions of potassium hydroxide in the hope that any organic fat present would be saponified and that this might serve as a histo-chemical method for identifying paraffin, since the latter is not saponifiable. Iodine, Sudan III, Nile blue sulphate, the Smith Dietrich osmic acid method, and Ziehl-Nielsen 's stain were used in the staining of frozen sections. All sections were compared with sections of an endogenous lipoid pneumonia in a case of carcinoma of the bronchus.

The first and most useful distinguishing feature was observed during the manipulation of the frozen sections, when it was found that clearly visible oil globules rose rapidly to the surface of the water when the sections were washed. No such globules were seen in the case of endogenous lipoid pneumonia.

The substance of the lung was mainly solid and contained few aerated spaces. It presented a mixed pattern of alveolar consolidation, oedema, interstitial pneumonia, and lobular collapse (Figs. 3, 4, 5). The cellular exudate in the alveolar spaces consisted of oilladen macrophages lying discretely or forming irregular compact casts (Fig. 3). These macrophages varied considerably in size, some being no larger than an average leucocyte, while at the other extreme there were very large cells with distorted outlines filled with numerous coarse globules of oil (Fig. 5). Large oil globules were also seen lying free in some alveoli and bronchioles (Fig. 4). All these globules stained with Sudan III, but the attempt to stain the paraffin specifically proved unsuccessful. The large free globules of oil stained blue with Nile blue sulphate and dark brown to black with osmic acid. This staining was denser and darker towards the periphery of the globules and lighter in the centre. The small intracellular globules of oil stained well with Sudan III, and faintly, or not at all, with the other dyes. All the globules were insoluble in absolute alcohol and soluble in xylol and chloroform. The attempt to saponify the fat in the control sections from the endogenous lipoid pneumonia with potassium hydroxide leaving the oil intact in the sections taken from the present case was unsuccessful. Solutions of potassium hydroxide which were sufficiently concentrated to saponify the fats invariably destroyed the tissues. Ziehl-Nielsen's stain showed no acid-fast material.

In some areas the alveoli were filled with serous fluid and contained no cellular exudate. The alveolar septa in such areas had dilated capillaries but little or no inflammatory infiltration. This appearance was in contrast with other areas where interstitial pneumonia was marked and the alveolar spaces almost completely obliterated by the expansion of the swollen septa. The 
cellular exudate in such septa consisted of some oil-laden macrophages, numerous histiocytes containing a varying amount of cytoplasm but no visible oil, and lymphocytes. Polymorphonuclear leucocytes were scarce.

Some catarrhal desquamation of the epithelium was seen in the bronchioles and many of them also contained oil macrophages. Many of the bronchioles showed chronic inflammatory changes with plication of the mucosa and reduction or complete occlusion of the lumen.

The regional lymph nodes were slightly oedematous, congested and showed a moderate amount of sinus catarrh. A few oil macrophages were seen in the sinuses of these nodes.

\section{Discussion}

Pathology. The histological features of the oil pneumonias are well known. They have been fully investigated by Pinkerton (1927 and 1928), Ikeda (1937), Paterson (1938), and Graef (1939) in human necropsies and animal experiments. The lung responds to the inhalation of oil by a foreign body reaction, with an outpouring of macrophages into the alveoli, and by the later formation of giant cells and septal fibrosis. The nature of the aspirated oil can to some extent be judged from the histological appearance. Fats with a high free fatty acid content or fats which are rapidly hydrolysed tend to produce an acute inflammatory process with oedema and necrosis. This is the case with all animal fats and some vegetable fats such as chaulmoogra oil, peanut oil, and some samples of olive oil. Other vegetable oils, however, such as the iodized poppy seed oil and castor oil, do not appear to be irritating to the lung and seem to be removed by expectoration producing no necrosis and little or no foreign body reaction.

Inhalation of medicinal paraffin is characterized by its rapid emulsification and phagocytosis and the absence of acute necrosis. The alveoli become filled with mononuclear cells containing fine droplets of paraffin, a patchy or confluent area of consolidation being produced. The septa are engorged with macrophages, histiocytes and lymphocytes and are later invaded by fibroblasts. The oil appears to be removed with difficulty as it cannot be metabolized by tissue enzymes. As fibrosis proceeds the fine oil globules tend to coalesce to form larger droplets and the macrophages disintegrate. Ultimately a tumourlike lesion may be formed. These 'paraffinomata' are a feature of adult aspiration pneumonia.

If the quantity of oil inhaled is large or its rate of introduction rapid, much of it will remain unemulsified and large globules will be found in the air passages. The histological picture will be more of patchy and variable alveolar consolidation with lobular collapse and less of inflammatory interstitial infiltration or fibrosis. Macrophages containing oil droplets are also found in association with suppurative pneumonia and carcinoma of the bronchus, but the following features clearly demonstrated in our case helped to differentiate the condition from an endogenous lipoid pneumonia: (1) the presence of free large oil globules in the air passages; (2) the considerable irregularity of size and form of the paraffin oil macrophages; (3) the coarseness and variation in size of the intracellular oil globules; (4) the rise of oil globules to the surface on immersion of the lung or frozen sections of it in water.

While the irregularity in size of fat droplets and macrophages is not uncommon in cases of endogenous lipoid pneumonia and oil globules are sometimes seen to rise to the surface when other very fatty material such as lipomatous tissue is immersed in water, numerous free large globules of oil in the air passages are unlikely in any condition other than exogenous lipoid pneumonia.

Differential staining methods have received much attention and are of most value in distinguishing medicinal paraffin from other oils. While paraffin will stain with Sudan III it cannot, of course, reduce osmic acid as is the case with animal or vegetable oils containing even very small quantities of free unsaturated fatty acids. But differential staining methods may prove equivocal because paraffin is an excellent solvent of organic material and will absorb and adsorb such substances as albumen, mucus, steroids, and organic fats.

A curious reaction peculiar to cod liver oil discovered by Pinkerton (1928) is the formation of a membrane round the droplets which renders them insoluble in ordinary fat solvents. This membrane area stained by Ziehl-Nielson's method is acid-fast, and this process is a valuable means of identification.

The most satisfactory proof of the existence of liquid paraffin in an oil aspiration pneumonia, and one supplied in only a small proportion of the reported cases, is provided by chemical extraction and analysis of the suspect lung. This was first carried out by Fischer-Wasels (1933) and subsequently by Paterson (1938), Graef (1939), Kaplan (1941), and others.

In the case of other oils chemical extraction is not so useful but will give an idea of the excess of saponifiable fat over the average normal figures. The total fat content of the portion of the lung we examined was not estimated.

It is clear that liquid paraffin was the oil mainly responsible for the pneumonia in our case, but the finding in the air passages of large oil droplets staining with osmic acid suggests that a terminal aspiration of food fats may have occurred. 
Aetiology. It is well established that small quantities of oil introduced into the nose may find their way into the lungs. This has been demonstrated in animal experiments by Walsh and Cannon (1938) and in man by Quinn and Meyer (1929) who injected iodized oil into the nostrils of sleeping patients and were able, the next moming, to demonstrate its presence in the lung radiologically. Moreover, bronchography can be satisfactorily carried out by the injection of lipiodol into the nasopharynx of a patient under general or local anaesthesia.

Medicinal paraffin seems to be non-irritant to respiratory mucous membrane, and therefore its entry into the trachea may provoke no cough reflex even in a conscious patient, and Proetz (1934) has shown in animal experiments that whereas rabbit nasal ciliae continue to move indefinitely under a layer of liquid paraffin their 'streaming' action is feeble or absent.

In many reports of paraffin pneumonia in adults and children the oil has been introduced in the form of nose drops or throat sprays. The danger of this form of medication is therefore widely recognized. The National formulary appends a warning against the 'prolonged and continuous' use of oily nose drops. The circumstances in which their use is dangerous is, however, less clear. First, excessive dosage predisposes to aspiration as exemplified in reports of adult victims of oil pneumonia whose habit it had been to give themselves daily nasal douches of medicinal paraffin as in the third case reported by Davis (1936). Secondly the position of the head at the time of instillation or spraying may play a part, Griesman (1944) having shown in experimental studies that the danger is greatest if the head is extended. Thirdly, factors described below which tend to increase the chance of aspiration of oils administered by mouth probably play a similar role in the case of nose drops or sprays. That oil given by mouth should enter the lungs seems hard to credit, but there are now many reports of the finding of exogenous oil pneumonia in patients to whom the oil had been administered orally.

The victims can be considered in two categeries. A large minority of the reported cases have occurred in infants; the remainder have occurred in individuals who have been suffering from chronic immobilizing diseases such as hemiplegia or rheumatoid arthritis involving prolonged decubitus and constipation, or who have had local or neurogenic diseases which have resulted in dysphagia. Because of the nature of the predisposing diseases most of this latter group of patients have been middle-aged or elderly, although cases in children and young adults have been reported.
In the cases of oil aspiration pneumonia in infants the oil involved has been in the great majority of cases medicinal paraffin given nasally or orally, or cod liver oil by mouth. (In the remainder milk fat. or egg yolk, have been incriminated, but proof of the presence of these food fats in the lungs is very difficult to obtain.)

It is not easy to judge from the literature the relative dangers of nasal or oral administration of medicinal paraffin to infants. In many cases both routes have been involved and in many others it is not clearly stated that the oil was given only by the one or the other route. However, it is clear, both from the cases of paraffin pneumonia like our own in which nose drops were not used, and from the frequently reported cases of cod liver oil pneumonia, that oral administration alone may result in aspiration.

It is aetiologically significant that practically none of the affected infants was in good health before the administration of oil. The morbid conditions most frequently found were chronic upper respiratory infections with nasal obstruction and chronic feeding difficulties associated with vomiting or malnutrition. But, in addition, a large proportion of the infants were also the victims of congenital disorders, either interfering directly with deglution, as for instance in cases of cleft palate, or resulting in relative immobility or debility. Congenital cerebral palsy, mongolism, meningocele, and congenital heart disease are amongst some of the associated conditions.

The danger of inhalation pneumonia in premature infants is widely recognized. It is therefore not surprising to find recorded cases of fatal aspiration pneumonia in premature infants, in the causation of which the aspiration of cod liver oil or medicinal paraffin appears to have played a major role. Frequently the infants have resisted the administration of the oil and choking has resulted.

In one case (Monfort, 1939), a 7-weeks-old child in good health was forcibly given mineral oil whilst crying, immediately developing dyspnoea and a severe barking cough. A radiograph showed extensive bilateral hilar infiltration. In a case described by Cannon (1935) a 5-month-old boy was found at necropsy to have the typical features of lipoid pneumonia with secondary lung abscesses containing mixed organisms. He had developed a cough shortly after the first administration of cod liver oil at the age of 1 month, the dose being given whilst the child lay on his back. Subsequent doses were resisted and the oil was forced on him while his nose was held.

One may mention the theoretical possibility that paraffin oil might reach the lungs not by aspiration 
but via the intestine and blood stream. Frazer, Schulman, and Stewart (1944) have shown that finely dispersed paraffin emulsion may be absorbed from the rat's intestine in particulate form, but they emphasize that such an emulsion does not normally occur when paraffin is administered to man. If, however, this were the case, the oil particles would pass via the lacteals and thoracic duct into the venous system and thence to the right heart and lungs.

Policard (1941) in a paper which does not give experimental results in detail, states that if oil is injected intraperitoneally in rabbits and guinea-pigs, it rapidly finds its way to the lungs, where it produces an "alveolitis "indistinguishable from that of lipoid pneumonia.

Busquet and Vischniac (1921) injected olive oil into the saphenous veins of dogs and concluded by chemical examination that the greater part of the injected oil came to rest in the lungs within a short period. To obviate the possibility that the oil might be arrested simply at the first capillarv filter, they repeated the experiment injecting the oil into the portal vein and again found a much higher percentage in the lungs than in the liver.

The chain of evidence for the theory of intestinal absorption is indeed tenuous. Evidence in favour of the aspiration theory is provided by the fact that the oil in the lungs usually shows the foreign body type of distribution. Goodwin (1934) points out that in the reported cases in infants the right lung is affected more than the left, the upper lobes more than the lower, and the posterior segments more than the anterior. A foreign body distribution was also observed by Freiman, Engelberg, and Merrit (1940) in their review of a personal series of 47 cases of exogenous oil pneumonia in adults. In our case the oil was fairly evenly distributed throughout both lungs and in all lobes, but by analogy with other cases aspiration was almost certainly the mode of entry.

The total weight of the wet lungs in our patient was $121 \mathrm{~g}$. Of this a representative portion weighing $7 \mathrm{~g}$. was extracted and from this $0 \cdot 800 \mathrm{~g}$. of paraffin was obtained. The whole lung must, therefore, have contained something of the order of $13.8 \mathrm{~g}$. or $16 \mathrm{ml}$. of medicinal paraffin, that is to say, a little more than one day's dose. This, combined with the fact that in some of the recorded cases oil has been administered on only a few occasions, e.g., Laughlen`s case III (1925). Pinkerton's case I (1927) suggests that the more normal prolonged dosage may simply increase the chances of occasional aspiration taking place rather than allowing a continual slow accumulation of oil in the lungs.
Can oil aspiration pneumonia in itself cause death ? In most of the published reports in which full post-mortem details are given some additional infection seems to have immediately preceded death. A superimposed purulent pneumonia is the commonest; meningitis, mastoiditis, and pyelonephritis are amongst the other conditions mentioned. It is obvious, however, that when the lungs are 'oillogged' extensively as in the case described here, a very slight pulmonary infection will be sufficient to kill the child.

Diagnosis and Treatment. In the great majority of reported cases, including ours, the diagnosis was not made in life. Proof of the diagnosis in an infant must be almost impossible to obtain before death, but the history of prolonged or excessive adniinistration of medicinal paraffin or cod liver oil to a debilitated infant or to one who actively resists dosing, should in the presence of symptoms or signs of pneumonia suggest this diagnosis. It has been pointed out by Pierson (1932) that radiographs may reveal much more extensive consolidation than the clinical examination would suggest, but the radiograph of our case gave little idea of the extent of the infiltration as discovered at necropsy. Shadows shown radiographically tend to be maximal in the perihilar region, and, as Goodwin (1934) has shown, if the patient survives the normal lung appears to grow peripherally leaving the affected portions centrally, but it is characteristic that the radiological appearance changes very slowly.

Biopsy recommended as a diagnostic measure in adults by Nathanson, Frenkel, and Jacobi (1943) is hardly applicable to infants. It is, of course, difficult to obtain sputum from an infant, especially in quantities large enough to allow identification of the oil and thus to exclude an endogenous lipoid pneumonia in which lipophages may sometimes be expectorated.

The only possible treatment of the established condition is to stop the administration of the suspected oil and to guard against, and where necessary treat, secondary infections.

Prophylaxis. (1) The dangers of medicinal paraffin nose drops probably outweigh any advantages they may have over aqueous preparations and should therefore not be used. (2) Oesophageal or gastric tubes, bronchoscopes, etc., should not be lubricated with medicinal paraffin. (3) Medicinal paraffin is administered so frequently by mouth without apparent harmful effects, that the danger of oil pneumonia in a healthy infant must be considered remote. However, its use is contraindicated in infants who resist oil administration, who are suffering from dysphagia or cough, or who are debilitated as the result of illness or congenital 
deformity. In such cases, if constipation cannot be corrected by dietary measures, aperients other than liquid paraffin should be used. (4) These warnings also apply to the administration of cod liver oil. In those circumstances it is preferable to administer synthetic vitamin concentrates of which prophylactic doses can be given in smaller volumes.

\section{Summary}

The history of the recognition of oil aspiration pneumonia is reviewed.

A case of fatal paraffin pneumonia in an infant of $8 \frac{1}{2}$ months is described from the clinical and pathological standpoints.

The aetiology of the condition in infants and its prevention are discussed.

Our thanks are due to Dr. Reginald Lightwood for permission to publish this case, and to him and to Professor W. D. Newcomb for their criticism and advice; to Professor R. T. Williams and Dr. D. V. Parke for their co-operation and assistance with the chemical investigation; to Mr. A. E. Beasley for the preparation of the histological sections; to Dr. P. N. Cardew for the photographs, and to Dr. K. W. Cross for his helpful suggestions.

\section{REFERENCES}

Bishop, P. G. C. (1940). Ann. intern. Med., 13, 132?. Busquet, H., and Vischniac, C. (1921). C.R. Soc. Biol., Paris, 84, 852.
Cannon, P. R. (1935). Trans. Chicago path. Soc., 14, 219.

Corper, H. J., and Freed, H. (1922). J. Amer. med. Ass., 79. 1739.

Davis, K. S. (1936). Radiology, 26, 131.

Fischer-Wasels. B. (1933). Frankfurt. Z. Path., 44, 412.

Frazer, A. C., Schulman, J. H., and Stewart, H. C. (1944). J. Physiol., Lond., 103, 306.

Freiman, D. G., Engelberg. H., and Merrit, W. H. (1940). Arch. intern. Med., 66, 11.

Goodwin, T. C. (1934). Amer. J. Dis. Child., 48, 309.

Graef, I. (1939). Arch. Path., 28, 613.

Griesman, B. L. (1944). Arch. Otolaryng., Chicago, 39, 124.

Guieysse-Pellissier, A. (1920). C.R. Soc. Biol., Paris, 83, 809.

Ikeda, K. (1935). Amer. J. Dis. Child., 49, 985. (1937). Arch. Path., 23, 470.

Kaplan, L. (1941). Amer. J. Dis. Child., 62, 1217.

Laughlen, G. F. (1925). Amer. J. Path., 1, 407.

Monfort (1939). Med. Times, N.Y., 67, 320.

Nathanson, L., Frenkel, D., and Jacobi, M. (1943). Arch. intern. Med., 72, 627.

Paterson, J. L. H. (1938). J. Path. Bact., 46, 151.

Pierson, J. W. (1932). Amer. J. Roentgenol., 27, 572.

Pinkerton, H. (1927). Amer. J. Dis. Child., 33, 259. (1928). Arch. Path., 5, 380.

Policard, A. (1941). C.R. Soc. Biol., Paris, 135, 1072.

Proetz, A. W. (1934). J. Laryng., 49, 557.

Quinn, L. H., and Meyer, O. O. (1929). Arch. Otolaryng., Chicago, 10, 152.

Walsh, T. E., and Cannon, P. R. (1938). Ann. Otol., etc., St. Louis, 47, 579.

Waters, C. A., Bayne-Jones, S., and Rowntree, L. G. (1917). Arch. intern. Med., 19, 538. 